\title{
A Solution with Bluetooth Low Energy Technology to Support Oral HealthCare Decisions for Improving Oral Hygiene
}

\author{
Aeddula Omsri Kumar \\ Blekinge Institute of Technology, \\ Sweden \\ Anderberg Peter \\ Blekinge Institute of Technology, \\ Sweden
}

\author{
Flyborg Johan \\ Blekinge Institute of Technology, \\ Sweden \\ Berglund Johan Sanmartin \\ Blekinge Institute of Technology, \\ Sweden
}

\author{
Larsson Tobias \\ Blekinge Institute of Technology, \\ Sweden \\ Renvert Stefan \\ Kristianstad University, Sweden
}

\begin{abstract}
The advent of powered toothbrushes and associated mobile health applications provides an opportunity to collect and monitor the data, however collecting reliable and standardized data from large populations has been associated with efforts from the participants and researchers. Finding a way to collect data autonomously and without the need for cooperation imparts potentiality to build large knowledge banks. A solution with Bluetooth low energy technology is designed to pair a powered toothbrush with a single-core processor to collect raw data in a real-time scenario, eliminating the manual transfer of powered toothbrush data with the mobile health applications. Associating powered toothbrush with a singlecore processor is believed to provide reliable and comprehensible data of toothbrush use and propensities can be a guide to improve individual exhortation and general plans on oral hygiene quantifies that can prompt improved oral wellbeing. The method makes a case for an expanded chance to plan assistant capacities to protect or improve factors that influence oral wellbeing in individuals with mild cognitive impairment. The proposed framework assists with determining various parameters, which makes it adaptable and conceivable to execute in various oral care contexts.
\end{abstract}

\section{CCS CONCEPTS}

- Applied Computing; • Life and medical sciences; • Health care information systems;

\section{KEYWORDS}

Dental device, Oral health, Oral hygiene, Oral health information system

\section{ACM Reference Format:}

Aeddula Omsri Kumar, Flyborg Johan, Larsson Tobias, Anderberg Peter, Berglund Johan Sanmartin, and Renvert Stefan. 2021. A Solution with Bluetooth Low Energy Technology to Support Oral HealthCare Decisions for Improving Oral Hygiene. In 2021 5th International Conference on Medical and Health Informatics (ICMHI 2021), May 14-16, 2021, Kyoto, Japan. ACM, New York, NY, USA, 6 pages. https://doi.org/10.1145/3472813.3473179

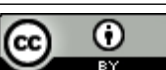

This work is licensed under a Creative Commons Attribution International 4.0 License.

ICMHI 2021, May 14-16, 2021, Kyoto, Japan

(C) 2021 Copyright held by the owner/author(s).

ACM ISBN 978-1-4503-8984-6/21/05.

https://doi.org/10.1145/3472813.3473179

\section{INTRODUCTION}

Life expectancy has increased over the past century due to major medical research results such as the discovery of antibiotics, various vaccines, and also social policy work for a better standard of living for the population [1]. As a result, the prevalence of individuals with mild cognitive impairments (MCI) and dementia has also increased [2]. It is well documented that cognitive impairment and dementia lead to an increased incidence of oral diseases, resulting in rapid deterioration of oral health and quality of life (QoL) [3], [4]. At the same time more elderly people in Sweden, as in many other higher-income countries, retain their teeth for a longer period of life [5]. The oral hygiene management of people suffering from cognitive impairment and dementia is critical and important to maintain good oral health as an integral part of their general health [6]. Oral hygiene constitutes the primary preventive measure of oral disease, and there exists a connection between oral hygiene and QoL in older adults [7]. Decreasing oral health in this group leads to individual suffering and increasing financial and resource demands on society. Finding aids to maintain and improve good oral hygiene for people with MCI and dementia is of great importance to reduce pain and illness and maintain a high level of Quality of life [8].

Information and communication technology (ICTs) have led to many significant transformations in the health care systems, particularly mobile health applications that have improved the quality of services provided to the people [9]. Patil et al., underlined mobile health application's effectiveness for improving oral hygiene and oral health outcomes in orthodontic patients [10]. The widespread popularity of mobile health applications for wireless devices provides an opportunity to influence people's behavior [11]. Accordingly, Oral-B [Procter \& Gamble, Cincinnati, OH, United States] has combined state-of-the-art wireless technology with clinically proven powered toothbrushes in an innovative mobile platform to increase cleaning performance, increase patient motivation, and create better daily oral hygiene habits through real-time instant feedback and tracking. The interactive Oral-B®Professional Care $6000{ }^{\circledR}$ with Bluetooth ${ }^{\circledR} 4.0$ connectivity allows for two-way communication between the toothbrush and the mobile application to enable instant feedback around brushing force and session length [12]. The limitation of the existing model is the limited storage capacity. This entails linking the powered toothbrush with the mobile health application, frequently transferring the data between the powered toothbrush and the mobile health application. This can be difficult to carry out both motorically and cognitively for 
elderly and sick individuals. Lack of continuity in the data transmission leads to loss of data and impairs usability. The existing model and its associated mobile application compiles and present a few possible parameters such as brush session length and brush power limits access to obtain more information from the powered toothbrush useful for decision-making. To register individuals', use of the powered toothbrush and, constructing from that, prepare individual oral hygiene advice and instructions and follow up on the results, extended functionality is required. It shall not require active manual data transfer and must present data regarding date, time, average brushing time and number of brushings per selected period. Collecting and storing data on oral health and oral hygiene in modern information systems provides knowledge and the opportunity to develop aids and methods for improved general and oral health, resulting in increased quality of life. Health care information systems can discover the required data about oral health and provide support in the healthcare decision-making process [13].

In this paper, a solution with Bluetooth low energy technology system is proposed to support oral healthcare decisions for improving oral hygiene will be explained and discussed. The remainder of this paper is organized as follows; In part 2, the proposed system is presented. In part 3 the proposed method is exemplified, and the paper concludes with discussion, conclusions, and directions for future work.

\section{MATERIAL AND METHODOLOGY}

\subsection{System Architecture}

The proposed system comprises two main components: hardware, and software. Integrating both the components supports in accomplishing the data acquisition process. Hardware components are essential for providing a medium for communication, and for the data gathering process, whereas software components are essential for establishing one-way data transfer between the hardware components.

2.1.1 Hardware Component. The hardware components consist of a powered toothbrush and a single-core processor. In the study, Oral-B®Professional Care $6000{ }^{\circledR}$ was used as a powered toothbrush and Raspberry Pi Zero W (RPiZ) was used as a single-core processor. Oral-B®Professional Care $6000{ }^{\circledR}$ is a rechargeable powered toothbrush with Bluetooth connectivity, which allows for connection to the smartphone to store the brushing data [14]. Raspberry Pi Zero $\mathrm{W}$ is a single-core processor extending the family of pi with added Bluetooth connectivity [15].

Powered Toothbrush. The study was carried out with Oral$\mathrm{B} \circledast$ Professional Care $6000{ }^{\circledR}$ as the powered toothbrush, however, the proposed system works for any powered toothbrush with Bluetooth connectivity feature. The employed powered toothbrush transmits or advertises data such as average brushing time, and a total time of applied pressure from the pressure sensor through Bluetooth packets. Each powered toothbrush contains a universally unique identifier (UUID) [14]. The transmitted data or the real-time data can be visualized in the developed Oral-B mobile application, however, each version of the mobile application differs in the amount of visualized data.
Single-core Processor. Raspberry Pi Zero W is a single-core processor, popularly known as a minicomputer extending the original Pi family with added functionalities such as Bluetooth connectivity, and wireless LAN. It requires a $1.2 \mathrm{~A}$ power supply and can be supplied using a micro USB charging case and consists of Bluetooth low energy technology and the other functionalities as the original raspberry pi. The single-core processor needs to flash write with a separate raspberry pi operating system and the system works on Linux operating environment [15]. A python programming in a Linux environment with the library modules supports a one-way data transmission process in a real-time scenario.

2.1.2 Software Component. The software component contains four main sub-components namely Bluetooth low energy, generic attribute profile (GATT), bluepy, and UUID in the testing process. As mentioned above, the main functionality of this component is to establish a connection between the hardware components. The data transmission mode for the system is initialized using the Bluetooth low energy connectivity. While Bluetooth low energy devices essentially communicate on a generic attribute profile (GATT). A python library module bluepy supports the process of initiating the GATT communication.

Bluetooth Low Energy. Bluetooth low energy is a low power wireless communication technology for control and monitoring applications, that can be used for shorter distance communications enabling smart devices to receive or transmit information [16]. $\mathrm{RPiZ}$ communicates with a powered toothbrush through Bluetooth technology.

Generic Attribute Profile. A GATT communication profile defines the way a powered toothbrush transmit or advertise data packets to RPiZ. GATT works under the concept of server and client relationship, Information uncovered by a server is introduced in a GATT profile which is a progressive design of traits permitting the exchange of data among server and client [16]. where RPiZ acts as the GATT server, which holds the attribute protocol lookup data and power toothbrush acts as GATT client, which sends data to the server.

Bluepy. Bluepy is the python module for establishing communications with Bluetooth low energy devices built on raspberry pi, internally working on the BlueZ library. BlueZ is an official Linux Bluetooth stack that provides support for core Bluetooth layers and protocols. The module consists of a peripheral, scanner, scan entry, default delegate, UUID, service, and characteristics classes for specific communication functionalities [17]. A peripheral class encapsulates a connection to Bluetooth low energy peripheral, once the connection is established services and characteristics offered by powered toothbrush can be discovered and read. Scanner object scans the within-range available Bluetooth devices. The information obtained from scanner object Bluetooth devices can be obtained from the Scan entry class. UUID class is an object value given by the user to connect to the powered toothbrush. Service class represents a collection of characteristics and descriptors which are all related to one particular function of the peripheral. Bluetooth LE characteristic class represents a short data item that can be read or written [17]. 


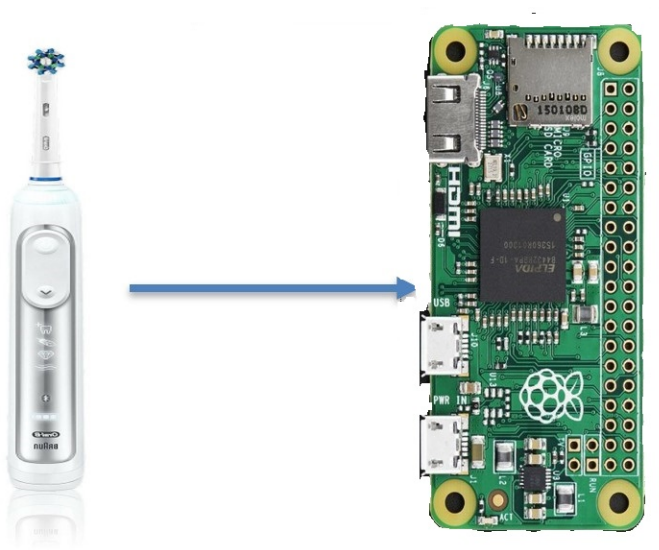

Figure 1: The proposed system.

UUID. A 128-bit UUID supports custom services and characteristics. As mentioned earlier, the UUID class is an object value given by the client to interface with the force toothbrush. "BLESCAN (Android)" or "LightBlue (IOS)" mobile application can be used to determine the UUID's of the powered toothbrush.

\subsection{Research Approach}

The research presented in this paper can be framed as one of the activities conducted during the Descriptive Study I phase of the Design Research Methodology (DRM) proposed by Blessing and Chakrabarti [18]. The methodology was based on the work [19, p. 6] focusing on the data transmission between Bluetooth low energy devices. In this respect, the usage of RPiZ was identified as a highly suitable method to determine various parameters, extending the opportunity to plan assistant abilities to protect or improve factors.

\subsection{Testing Process}

The participants are recruited from a sample of 170 individuals who participate in an ongoing study aimed to investigate the effect of introducing a powered toothbrush in people with MIC. Participants have undergone the Mini-Mental State Examination (MMSE) and have values between 20 and 28 [20]. They have also suffered from memory difficulties in the past six months. The participants were instructed in handling the powered toothbrush and recommended to use the brush for at least two minutes every morning and evening, with the system installed in their homes for a period of four to six months. Figure 1 shows the proposed system of communication where the brush data is transmitted to the RPiZ controller in a real-time scenario. The entire setup is executed in a python programming environment with suitable library modules. The Python module "bluepy" supports the process of initiating the GATT communication for data transmission. RPiZ is paired up with the specific power toothbrush using the UUID value or the raspberry pi zero scans locally for the powered toothbrush which advertises Bluetooth packets. Once the connection is established, the data from the powered toothbrush can be stored locally in the raspberry pi or network-based storage either as a text file or as a spreadsheet. The system is pre-installed with a virtual networking computing server to virtually operate the system at the participant's home and update the UUID of the powered toothbrush.

\section{RESULTS}

The proposed system is exemplified by installing the designed systems in the homes of the above-mentioned participants. Virtual network computing server assisted in associating the powered toothbrush with RPiZ using UUID. Associating the RPiZ with the powered toothbrush eliminates the problem of noisy data, where data from other powered toothbrushes here is referred to as noisy data. Figure 2 shows two instances of the proposed system installation in the participant's homes. The left part of Figure 2 shows

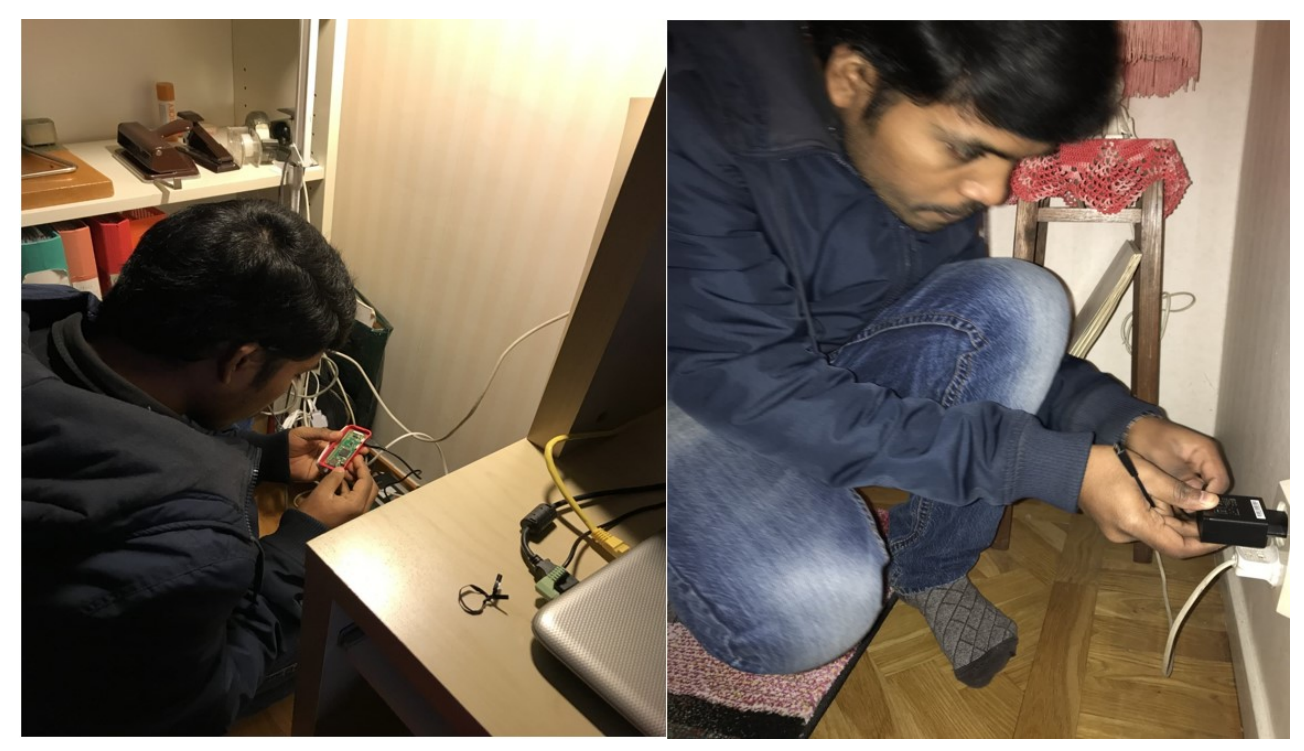

Figure 2: Installation of the system in the participant's home. 


\begin{abstract}
Date 2019-11-11 22:21:10.326789 Recorded time is 1 Secs Date 2019-11-11 22:21:11.739939 Recorded time is 2 Secs Date 2019-11-11 22:21:12.422798 Recorded time is 3 Secs Date 2019-11-11 22:21:13.348766 Recorded time is 4 Secs Date 2019-11-11 22:21:14.323982 Recorded time is 5 Secs Date 2019-11-11 22:21:15.494058 Recorded time is 6 Secs Date 2019-11-11 22:21:16.371408 Recorded time is 7 Secs Date 2019-11-11 22:21:17.493001 Recorded time is 8 Secs Date 2019-11-11 22:21:18.321510 Recorded time is 9 Secs Date 2019-11-11 22:21:19.345325 Recorded time is 10 Secs Date 2019-11-11 22:21:20.369093 Recorded time is 11 Secs Date 2019-11-11 22:21:21.441675 Recorded time is 12 Secs Date 2019-11-11 22:21:22.417302 Recorded time is 13 Secs Date 2019-11-11 22:21:23.343127 Recorded time is 14 Secs
\end{abstract}

Figure 3: Text file data of a participant (raw data).

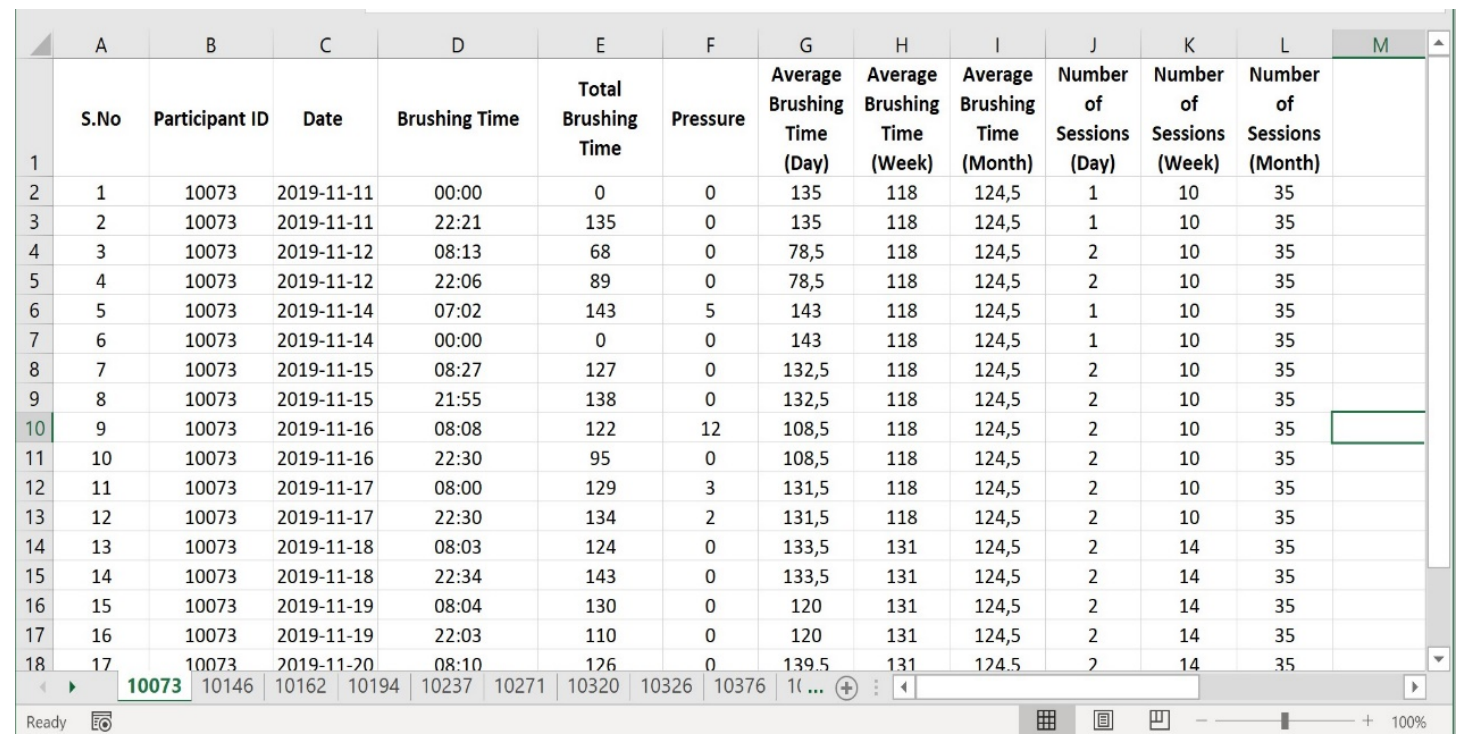

Figure 4: Spreadsheet file data of a participant.

raspberry pi zero $\mathrm{w}$ in a protective case, and the right part of Figure 2 shows the RPiZ connected to a power supply.

Figure 3 and Figure 4 show the text and a participant's spreadsheet format data, respectively. Text files represent raw data collected from the raspberry pi zero controller and the spreadsheet data is a processed data synthesized from raw data. The spreadsheet contains different estimated parameters as columns. The raw data from the text file was analyzed and transformed into meaningful information. The participant ID was a unique number assigned to each participant, and each assigned system name was replaced with the participant ID respectively. Python library module "DateTime" imports the date and time into the text file according to the brushing session, and the module "date finder" extracts the date and time from the text file [16]. These modules assist in extracting the date and time information. Extracting the digits between the strings helps to identify the total brushing time and similarly, the sensor 


\section{Total Brushing Time}

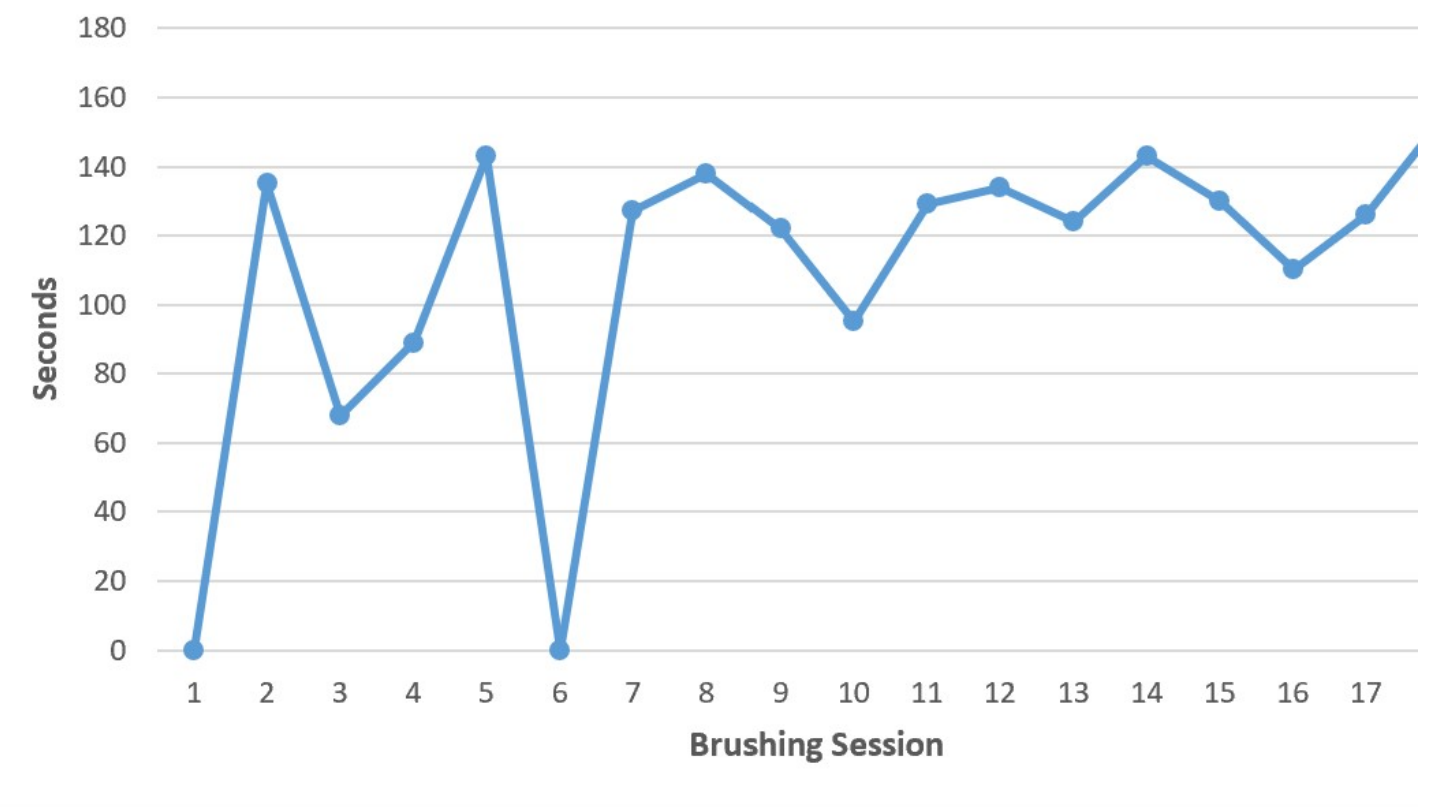

Figure 5: Data visualization of total brushing time.

data from the text was analyzed to determine the total applied pressure time per brushing session. These input parameters from the spreadsheet could be further used to determine other useful parameters such as average brushing time per day, week, month, and a total number of brushing sessions per day, week, month. Average brushing time per day was calculated based on an average of two rows in the spreadsheet and estimating the week number averaged the brushing time per week and similarly the average brushing time per month. The same logic applied to calculate the total number of sessions per day, week, and month. Different parameters could be visualized as useful for decision-making processes as shown in figure 5 and figure 6 . Figure 5 shows the visualization of the total brushing time of each session in seconds corresponding to participant ID 10073 and figure 6 shows the graph of average brushing time per day, week, and month with blue bar represents the average brushing time per day, orange and grey represents the average brushing time per week and per month respectively. Only one participant data with few days data was shown in the below figures for better understanding. These visualizations support knowledge about factors leading to good oral hygiene, help develop aids and improve oral and general health.

\section{DISCUSSION AND CONCLUSION}

The proposed system was designed to collect data from the powered toothbrush. The purpose was to create a base for increased opportunity to design auxiliary functions to preserve or improve factors that affect oral health in people with MCI. The proposed system was trained using a python environment involving library modules to collect the associated toothbrush data by establishing a GATT communication through Bluetooth connectivity between the powered toothbrush and single-core processor RPiZ. The methodology focuses on receiving and decoding the advertised Bluetooth packets to more meaningful information that is useful for the decision-making process to improve oral hygiene. The proposed system helps determine different parameters, making it flexible and possible to implement in different oral care contexts. The proposed method receives the advertised Bluetooth packets in real-time signifying that the powered toothbrush can be designed without having any memory slot. This provides the cost efficiency and simultaneously ensures the data transfer. Associating the UUID of the powered toothbrush ensures that obtained data would be noise-free. In conclusion, the ability to access reliable and comprehensive data on toothbrush use and habits can help improve individual advice and general plans on oral hygiene measures that can lead to improved oral health. Future works include integrating the features of the system with the powered toothbrush as a single usage product and providing a user interface for the proposed system to support users.

\section{ACKNOWLEDGMENTS}

The research leading to these results has received financial support by the Swedish Knowledge and Competence Development Foundation (Stiftelsen för kunskaps- och kompetensutveckling) through the Model Driven Development and Decision Support research profile at Blekinge Institute of Technology. 


\title{
Average Brushing Time
}

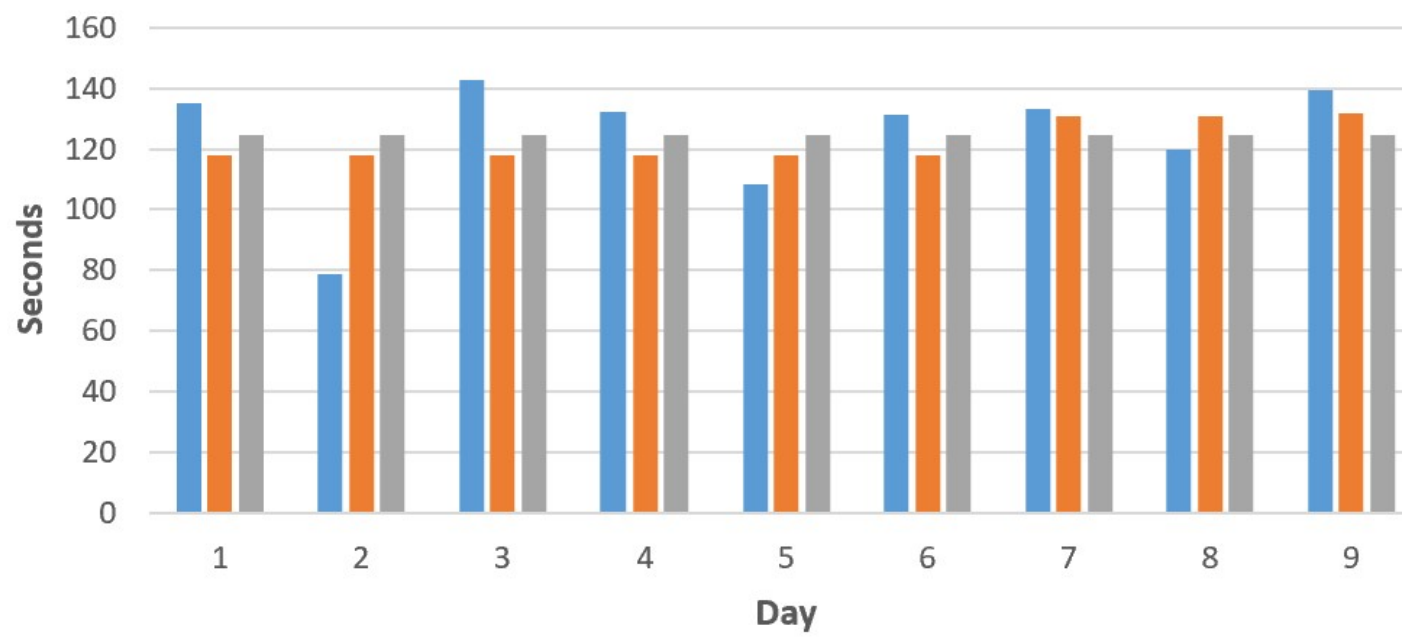

\author{
Average Brushing Time (Day) Average Brushing Time (Week) \\ Average Brushing Time (Month)
}

Figure 6: Data visualization of average brushing time.

\section{REFERENCES}

[1] E. M. Crimmins, 'Lifespan and Healthspan: Past, Present, and Promise', Gerontologist, vol. 55, no. 6, pp. 901-911, Dec. 2015, doi: 10.1093/geront/gnv130.

[2] M. Prince, R. Bryce, E. Albanese, A. Wimo, W. Ribeiro, and C. P. Ferri, 'The global prevalence of dementia: A systematic review and metaanalysis', Alzheimer's \& Dementia, vol. 9, no. 1, pp. 63-75.e2, Jan. 2013, doi: 10.1016/j.jalz.2012.11.007.

[3] S. Delwel, T. T. Binnekade, R. S. G. M. Perez, C. M. P. M. Hertogh, E. J. A. Scherder, and F. Lobbezoo, 'Oral health and orofacial pain in older people with dementia: a systematic review with focus on dental hard tissues', Clin Oral Investig, vol. 21, no. 1, pp. 17-32, Jan. 2017, doi: 10.1007/s00784-016-1934-9.

[4] L. J. M. van de Rijt et al., 'The Influence of Oral Health Factors on the Quality of Life in Older People: A Systematic Review', Gerontologist, vol. 60, no. 5, pp. e378-e394, Jul. 2020, doi: 10.1093/geront/gnz105.

[5] F. Schwendicke, J. Krois, T. Kocher, T. Hoffmann, W. Micheelis, and R. A. Jordan, "More teeth in more elderly: Periodontal treatment needs in Germany 1997-2030', J Clin Periodontol, vol. 45, no. 12, pp. 1400-1407, Dec. 2018, doi: $10.1111 /$ jcpe. 13020 .

[6] S. S. Gao, C. H. Chu, and F. Y. F. Young, 'Oral Health and Care for Elderly People with Alzheimer's Disease', Int J Environ Res Public Health, vol. 17, no. 16, Aug. 2020, doi: 10.3390/ijerph17165713.

[7] T. Willumsen, B. Fjaera, and H. Eide, 'Oral health-related quality of life in patients receiving home-care nursing: associations with aspects of dental status and xerostomia: Oral quality of life, teeth and saliva', Gerodontology, vol. 27, no. 4, pp. 251-257, Dec. 2010, doi: 10.1111/j.1741-2358.2009.00344.x.

[8] T. de S. Rolim et al., 'Evaluation of patients with Alzheimer's disease before and after dental treatment', Arq Neuropsiquiatr, vol. 72, no. 12, pp. 919-924, Dec. 2014, doi: 10.1590/0004-282X20140140.

[9] J. A. Powell, M. Darvell, and J. A. M. Gray, 'The Doctor, The Patient and the World-Wide Web: How the Internet is Changing Healthcare', J R Soc Med, vol. 96, no. 2, pp. 74-76, Feb. 2003, doi: 10.1177/014107680309600206.
[10] S. Patil et al., 'Effectiveness of mobile phone applications in improving oral hygiene care and outcomes in orthodontic patients', J Oral Biol Craniofac Res, vol. 11, no. 1, pp. 26-32, Mar. 2021, doi: 10.1016/j.jobcr.2020.11.004.

[11] B. Tiffany, P. Blasi, S. L. Catz, and J. B. McClure, 'Mobile Apps for Oral Health Promotion: Content Review and Heuristic Usability Analysis', JMIR Mhealth Uhealth, vol. 6, no. 9, p. e11432, Sep. 2018, doi: 10.2196/11432.

[12] C. Erbe et al., 'A comparative assessment of plaque removal and toothbrushing compliance between a manual and an interactive power toothbrush among adolescents: a single-center, single-blind randomized controlled trial', BMC Oral Health, vol. 18, no. 1, p. 130, Aug. 2018, doi: 10.1186/s12903-018-0588-1.

[13] P. E. Petersen, D. Bourgeois, D. Bratthall, and H. Ogawa, 'Oral health information systems-towards measuring progress in oral health promotion and disease prevention', Bull World Health Organ, vol. 83, no. 9, pp. 686-693, Sep. 2005, doi: /S0042-96862005000900014.

[14] 'Oral-B Genius 6000 Rechargeable Electric Toothbrush | Oral-B'. https://oralb. com/en-us/oral-b-genius-6000-rechargeable-electric-toothbrush/ (accessed Dec. 31, 2020).

[15] 'Buy a Raspberry Pi Zero W - Raspberry Pi'. https://www.raspberrypi.org/ products/raspberry-pi-zero-w/ (accessed Dec. 31, 2020).

[16] S. I. G. Bluetooth, 'Specification of the Bluetooth System-Covered Core Package version: 4.0', Bluetooth Special Interest Group, 2010.

[17] 'bluepy - a Bluetooth LE interface for Python - bluepy 0.9.11 documentation'. http://ianharvey.github.io/bluepy-doc/ (accessed Jan. 01, 2021).

[18] L. T. M. Blessing, A. Chakrabarti, and L. T. M. Blessing, DRM, a design research methodology. Dordrecht; London: Springer, 2009.

[19] J. Campos, S. Colteryahn, and K. Gagneja, 'IPv6 transmission over BLE Using Raspberry PI 3', in 2018 International Conference on Computing, Networking and Communications (ICNC), Maui, HI, Mar. 2018, pp. 200-204, doi: 10.1109/ICCNC.2018.8390350.

[20] M. F. Folstein, S. E. Folstein, and P. R. McHugh, "'Mini-mental state”, Journal of Psychiatric Research, vol. 12, no. 3, pp. 189-198, Nov. 1975, doi: 10.1016/00223956(75)90026-6. 